\title{
ON THE ORDER OF SOME ERROR FUNCTIONS RELATED TO $k$-FREE INTEGERS ${ }^{1}$
}

\author{
V. S. JOSHI
}

Abstract. Let $\Delta_{k}(x)$ and $\Delta_{k}^{\prime}(x)$ be the error functions in the asymptotic formulae for the number and the sum of $k$-free integers not exceeding $x$. We prove that on the assumption of Riemann hypothesis, we have

and

$$
\Delta_{k}^{\prime}(x)-x \Delta_{k}(x)=O\left(x^{1+1 / 2 k+\varepsilon}\right)
$$

$$
\frac{1}{x} \int_{1}^{x} \Delta_{k}(t) d t=O\left(x^{1 / 2 k+\varepsilon}\right)
$$

for arbitrary $\varepsilon>0$.

1. Introduction. Let $k$ be a fixed integer $\geqq 2$. A positive integer $n$ is called $k$-free if it is not divisible by the $k$ th power of any prime. We let $\mu_{k}$ denote the characteristic function of the set of $k$-free integers.

Almost throughout this paper, Riemann hypothesis is assumed to be true, $x$ denotes a positive real number and $\varepsilon$ is an arbitrary positive number. Also, $s=\sigma+i t$, where $\sigma$ and $t$ are real.

Let $Q_{k}(x)$ and $Q_{k}^{\prime}(x)$ denote the number and the sum of $k$-free integers not exceeding $x$, then it is well known that

$$
\Delta_{k}(x)=Q_{k}(x)-x / \zeta(k)
$$

and

$$
\Delta_{k}^{\prime}(x)=Q_{k}^{\prime}(x)-x^{2} /(2 \zeta(k)) .
$$

Vaidya [5] proved, using analytic methods, that on the Riemann hypothesis, we have

$$
\Delta_{2}^{\prime}(x)-x \Delta_{2}(x)=O\left(x^{1+1 / 4+\varepsilon}\right)
$$

and

$$
\frac{1}{x} \int_{1}^{x} \Delta_{2}(t) d t=O\left(x^{1 / 4+\varepsilon}\right)
$$

Received by the editors August 19, 1971.

AMS 1970 subject classifications. Primary $10 \mathrm{H} 15$.

Key words and phrases. $k$-free integers, Riemann hypothesis.

${ }^{1}$ This research was supported by UGC. 
D. Suryanarayana and R. Sitaramachandra Rao [2] proved by using only elementary methods that for a fixed integer $k \geqq 2$,

$$
\Delta_{k}^{\prime}(x)-x \Delta_{k}(x)=O\left(x^{1+3 /(4 k+1)+\varepsilon}\right)
$$

and

$$
\frac{1}{x} \int_{1}^{x} \Delta_{k}(t) d t=O\left(x^{3 /(4 k+1)+\varepsilon}\right) .
$$

In this paper, we follow Vaidya's method to prove that for any fixed integer $k \geqq 2$,

$$
\Delta_{k}^{\prime}(x)-x \Delta_{k}(x)=O\left(x^{1+1 / 2 k+\varepsilon}\right)
$$

and

$$
\frac{1}{x} \int_{1}^{x} \Delta_{k}(t) d t=O\left(x^{1 / 2 k+\varepsilon}\right) .
$$

We note that (1.7) and (1.8) are considerably stronger than (1.5) and (1.6). Of course, all these results depend on the Riemann hypothesis. We shall also see that the mistake in [5] which is noticed by the authors of [2], does not affect the final result presented by Vaidya in [5].

In $\S 2$, we list a number of theorems with proofs wherever necessary. These theorems are then used in $\S 3$ to prove (1.7) and (1.8).

2. THEOREM 1. If $\sigma>1(s=\sigma+i t)$,

$$
\sum_{n=1}^{\infty} \frac{\mu_{k}(n)}{n^{s}}=\frac{\zeta(s)}{\zeta(k s)} .
$$

Proof. Easy.

THEOREM 2 [3, p. 82]. If $0<\sigma<1$, then as $|t| \rightarrow \infty$

$$
\zeta(s)=O\left(|t|^{(1-\sigma) / 2}\right) \text {. }
$$

THEOREM 3 [3, p. 283]. If $\sigma>\frac{1}{2}$, then on the assumption of Riemann hypothesis, we have, as $|t| \rightarrow \infty$

$$
\frac{1}{\zeta(s)}=O\left(|t|^{\varepsilon}\right) .
$$

THEOREM 4 [5, p. 199].

$$
\begin{aligned}
\frac{1}{2 \pi i} \int_{\Sigma-i \infty}^{2+i \infty} \frac{x^{s}}{s(s+1) \cdots(s+m)} d s & =\frac{1}{m !}\left(1-\frac{1}{x}\right)^{m}, & & \text { if } x \geqq 1, \\
& =0, & & \text { if } 0<x<1 .
\end{aligned}
$$


For integers $m \geqq 0$, we define functions $G_{m}(x)$ by

$$
G_{m}(x)=\sum_{n \leqq x} \mu_{k}(n) n^{m}-\frac{x^{m+1}}{(m+1) \zeta(k)} .
$$

It is clear that if we write $G(x)$ for $G_{0}(x)$, then

$$
\Delta_{k}(x)=G(x)
$$

and

$$
\Delta_{k}^{\prime}(x)=G_{1}(x) .
$$

THEOREM 5. On the assumption of the Riemann hypothesis, we have for all integers $m \geqq 1$,

$$
(G-x)^{m}=O\left(x^{m+1 / 2 k+\varepsilon}\right),
$$

where on the left,$G^{r}$ is to be replaced by $G_{r}(x)$ after the binomial expansion.

Proof. In the result of Theorem 4 , we replace $x$ by $x / n$, multiply by $\mu_{k}(n)$ and add for $n=1$ to $n=\infty$. We get

$$
\sum_{n=1}^{\infty} \frac{1}{2 \pi i} \int_{2-i \infty}^{2+i \infty} \frac{\mu_{k}(n) x^{s} n^{-s}}{s(s+1) \cdots(s+m)} d s=\frac{x^{-m}}{m !} \sum_{n \leqq x} \mu_{k}(n)(x-n)^{m} .
$$

We first consider the right side of (2.4). We have

$$
\begin{aligned}
\sum_{n \leqq x} \mu_{k}(n)(x-n)^{m} & =(-1)^{m} \sum_{n \leqq x} \mu_{k}(n)(n-x)^{m} \\
& =\sum_{n \leqq x} \mu_{k}(n) \sum_{r=0}^{m}\left(\begin{array}{l}
m \\
r
\end{array}\right) n^{r} x^{m-r}(-1)^{r} \\
& =\sum_{r=0}^{m}(-1)^{r}\left(\begin{array}{c}
m \\
r
\end{array}\right) x^{m-r} \sum_{n \leqq x} \mu_{k}(n) n^{r} \\
& =\sum_{r=0}^{m}(-1)^{r}\left(\begin{array}{c}
m \\
r
\end{array}\right) x^{m-r}\left[G^{r}+\frac{x^{r+1}}{(r+1) \zeta(k)}\right] \\
& =\sum_{r=0}^{m}(-1)^{r}\left(\begin{array}{c}
m \\
r
\end{array}\right) x^{m-r} G^{r}+\sum_{r=0}^{m} \frac{(-1)^{r}\left(\begin{array}{c}
m \\
r
\end{array}\right) x^{m+1}}{(r+1) \zeta(k)} \\
& =(G-x)^{m}+\frac{x^{m+1}}{\zeta(k)} \sum_{r=0}^{m} \frac{(-1)^{r}}{(r+1)}\left(\begin{array}{c}
m \\
r
\end{array}\right) \\
& =(G-x)^{m}+\frac{x^{m+1}}{\zeta(k)(m+1)} \sum_{r=0}^{m}(-1)^{r}\left(\begin{array}{c}
m+1 \\
r+1
\end{array}\right) \\
& =(G-x)^{m}+\frac{x^{m+1}}{\zeta(k)(m+1)} \cdot
\end{aligned}
$$


Hence

$$
\frac{x^{-m}}{m !} \sum_{i=1} \mu_{k}(n)(x-n)^{m}=\frac{(G-x)^{m} x^{-m}}{m !}+\frac{x}{\zeta(k)(m+1) !} .
$$

We now consider the left side of (2.4). We first note (cf. [1] and [4]) that the order of summation and integration can be interchanged for the following reasons: If $f_{n}(s)$ denotes the integrand, then (i) for each $n, f_{n}(s)$ is continuous on the line $\sigma=2$, (ii) the series $\sum_{n=1}^{\infty} f_{n}(s)$ converges uniformly on any finite interval of the line $\sigma=2$, and (iii)

$$
\int_{-\infty}^{+\infty} \sum_{n=1}^{\infty}\left|\frac{\mu_{k}(n) x^{2+i t} n^{-2-i t}}{(2+i t)(3+i t) \cdots(m+2+i t)}\right| d t
$$

is convergent. (These assertions have been proved in a separate appendix, seen by the referee but not included for publication.) So changing the order of summation and integration and using Theorem 1, we see that the left side of (2.4) is

$$
\begin{aligned}
& \frac{1}{2 \pi i} \int_{2-i \propto}^{2+i \infty} \frac{x^{s \zeta(s)}}{\zeta(k s) s(s+1) \cdots(s+m)} d s \\
& \quad=\lim _{T \rightarrow \infty} \frac{1}{2 \pi i} \int_{2-i T}^{2+i T} \frac{x^{s \zeta} \zeta(s)}{\zeta(k s) s(s+1) \cdots(s+m)} d s .
\end{aligned}
$$

To evaluate this last integral, we consider the rectangle $\Gamma$ whose vertices are $A=2-i T, B=2+i T, C=1 / 2 k+\varepsilon+i T$ and $D=1 / 2 k+\varepsilon-i T$.

Now if we assume Riemann hypothesis, the integrand is regular within and on $\Gamma$ except for a simple pole at $s=1$, with residue $x /(m+1) ! \zeta(k)$. Therefore

$$
\frac{1}{2 \pi i} \int_{\Gamma} \cdots d s=\frac{x}{(m+1) ! \zeta(k)}
$$

i.e.

$$
\frac{1}{2 \pi i} \int_{A}^{B}+\int_{D}^{C}+\int_{C}^{D}+\int_{D}^{A}=\frac{x}{(m+1) ! \zeta(k)},
$$

or

$$
\begin{aligned}
\frac{1}{2 \pi i} \int_{A}^{\prime:} & =\frac{x}{(m+1) ! \zeta(k)}+\frac{1}{2 \pi i} \int_{C}^{I}+\frac{1}{2 \pi i} \int_{I)}^{(!}-\frac{1}{2 \pi i} \int_{D)}^{A} \\
& =\frac{x}{(m+1) ! \zeta(k)}+I_{1}+I_{2}-I_{3}, \quad \text { say. }
\end{aligned}
$$


Now

$$
\begin{aligned}
I_{1} & =\frac{1}{2 \pi i} \int_{1 / 2 k+\varepsilon+i T}^{2+i T} \frac{x^{s \zeta}(s)}{\zeta(k s) s(s+1) \cdots(s+m)} d s \\
& =O\left(x^{2} \int_{1 / 2 k+\varepsilon+i T}^{2+i T} \frac{\zeta(\sigma+i T)}{\zeta(k \sigma+i k T)(\sigma+i T) \cdots(\sigma+m+i T)} d \sigma\right) \\
& =O\left(x^{2} \int_{1 / 2 k+\varepsilon}^{2}|T|^{1 / 2-1 / 4 k+\varepsilon-m-1} d \sigma\right), \text { by Theorems } 2 \text { and 3. }
\end{aligned}
$$

\section{Hence}

$$
I_{1}=O(1), \quad \text { as } T \rightarrow \infty .
$$

Similarly

$$
I_{3}=O(1), \quad \text { as } T \rightarrow \infty \text {. }
$$

Now

$$
\begin{aligned}
I_{2} & =\frac{1}{2 \pi i} \int_{1 / 2 k+\varepsilon-i T}^{1 / 2 k+\varepsilon+i T} \frac{x^{s \zeta}(s)}{\zeta(k s) s(s+1) \cdots(s+m)} d s \\
& =\frac{1}{2 \pi i} \int_{-T}^{+T} \frac{x^{1 / 2 k+\varepsilon+i t \zeta\left(\frac{1}{2 k}+\varepsilon+i t\right)}}{\zeta\left(\frac{1}{2}+k \varepsilon+i k t\right)\left(\frac{1}{2 k}+\varepsilon+i t\right) \cdots\left(\frac{1}{2 k}+m+\varepsilon+i t\right)} d t \\
& =O\left(x^{1 / 2 k+\varepsilon} \int_{-T}^{+T} \frac{\zeta\left(\frac{1}{2 k}+\varepsilon+i t\right)}{\zeta\left(\frac{1}{2}+k \varepsilon+i k t\right) \cdots\left(\frac{1}{2 k}+m+\varepsilon+i t\right)} d t\right) \\
& =O\left(x^{1 / 2 k+\varepsilon}\left\{\int_{-T}^{-1}+\int_{-1}^{+1}+\int_{+1}^{+T}\right\}\right)
\end{aligned}
$$

i.e.

$$
I_{2}=O\left(x^{1 / 2 k+\varepsilon}\left\{I_{21}+I_{22}+I_{23}\right\}\right), \text { say. }
$$

It follows from Theorems 2 and 3 that

Hence, since $m \geqq 1$,

$$
I_{21}=O\left(\int_{-T}^{-1}|t|^{1 / 2-1 / 4 k+\varepsilon-m-1} d t\right)
$$

$$
I_{21}=O(1), \text { as } T \rightarrow \infty .
$$

Similarly

$$
I_{23}=O(1), \quad \text { as } T \rightarrow \infty .
$$


Again, using Theorems 2 and 3, we see that

$$
\begin{aligned}
I_{22} & =O\left(\int_{-1}^{+1} \frac{|t|^{1 / 2-1 / 4 k+\varepsilon}}{\left(\frac{1}{2 k}\right)\left(\frac{1}{2 k}+1\right) \cdots\left(\frac{1}{2 k}+m\right)} d t\right) \\
& =O\left(\frac{1}{m !} \int_{0}^{1} t^{1 / 2-1 / 4 k+\varepsilon} d t\right),
\end{aligned}
$$

i.e.

$$
I_{22}=O(1) \text {. }
$$

Thus, from (2.9), (2.10), (2.11) and (2.12), we get

$$
I_{2}=O\left(x^{1 / 2 k+\varepsilon}\right) \text {. }
$$

So finally, collecting (2.6), (2.7), (2.8) and (2.13) together, we have,

$$
\frac{1}{2 \pi i} \int_{2-i \infty}^{2+i \infty} \frac{x^{s} \zeta(s)}{\zeta(k s) s(s+1) \cdots(s+m)} d s
$$

$$
=\frac{x}{\zeta(k)(m+1) !}+O\left(x^{1 / 2 k+\varepsilon}\right)
$$

Now combining (2.4), (2.5) and (2.14), we get

whence finally,

$$
\begin{aligned}
\frac{x}{\zeta(k)(m+1) !}+ & O\left(x^{1 / 2 k+\varepsilon}\right) \\
& =\frac{(G-x)^{m} x^{-m}}{m !}+\frac{x}{\zeta(k)(m+1) !},
\end{aligned}
$$

$$
(G-x)^{m}=O\left(x^{m+1 / 2 k+\varepsilon}\right) .
$$

3. The proofs of (1.7) and (1.8). We put $m=1$ in (2.15) to get

$$
G_{1}(x)-x G(x)=O\left(x^{1+1 / 2 k+\varepsilon}\right)
$$

and this is precisely the statement (1.7) in view of (2.2) and (2.3). To prove (1.8), we need the following

LEMMA. If $x$ is an integer, then

$$
\sum_{n \leqq x} G(n)=-\left(G_{1}(x)-x G(x)\right)+G(x)+\frac{x}{2 \zeta(k)} .
$$


Proof.

$$
\begin{aligned}
\sum_{n \leqq x} G(n) & =\sum_{n \leqq x}\left(\sum_{m \leqq n} \mu_{k}(m)-\frac{n}{\zeta(k)}\right) \\
& =\sum_{n \leqq x}(x-n+1) \mu_{k}(n)-\frac{x(x+1)}{2 \zeta(k)} \\
& =\sum_{n \leqq x}(x+1) \mu_{k}(n)-\sum_{n \leqq x} n \mu_{k}(n)-\frac{x(x+1)}{2 \zeta(k)} \\
& =(x+1)\left(G(x)+\frac{x}{\zeta(k)}\right)-\left(G_{1}(x)-\frac{x^{2}}{2 \zeta(k)}\right) \\
& =-\left(G_{1}(x)-x G(x)\right)+G(x)+\frac{x}{2 \zeta(k)} .
\end{aligned}
$$

We now prove (1.8). We have

$$
\begin{aligned}
\sum_{n \leqq x} n \mu_{k}(n) & =\sum_{n \leqq x} n\left(Q_{k}(n)-Q_{k}(n-1)\right) \\
& =-\sum_{n \leqq x} Q_{k}(n)(n-(n-1))+x Q_{k}(x) \\
& =-\sum_{n \leqq x} \int_{t-1}^{t} Q_{k}(t) d t+x G(x)+\frac{x^{2}}{\zeta(k)} \\
& =-\int_{1}^{x} Q_{k}(t) d t+x G(x)+\frac{x^{2}}{\zeta(k)} \\
& =-\int_{1}^{x}\left(G(t)+\frac{t}{\zeta(k)}\right) d t+x G(x)+\frac{x^{2}}{\zeta(k)} \\
& =-\int_{1}^{x} G(t) d t-\frac{x^{2}}{2 \zeta(k)}+O(1)+x G(x)+\frac{x^{2}}{\zeta(k)}
\end{aligned}
$$

Therefore

$$
\sum_{n \leqq x} n \mu_{k}(n)-\frac{x^{2}}{2 \zeta(k)}-x G(x)=-\int_{1}^{x} G(t) d t+O(1),
$$

i.e.

$$
G_{1}(x)-x G(x)=-\int_{1}^{x} G(t) d t+O(1) .
$$

So (3.2) and (3.1) clearly imply (1.8).

We reiterate that our proofs of (1.7) and (1.8) are valid only on the assumption of Riemann hypothesis.

I am indebted to Dr. A. M. Vaidya for his encouragement and valuable help during the preparation of this paper. I also wish to thank the referee for his valuable suggestions. 


\section{REFERENCES}

1. E. Hille, Analysis. Vol. I, Blaisdell, Waltham, Mass., 1964. Theorem B.2.3, p. 333.

2. D. Suryanarayana and R. Sitaramachandra Rao, On the order of the error function of the $k$-free integers, Proc. Amer. Math. Soc. 28 (1971), 53-58. MR 42 \#5927.

3. E. C. Titchmarsh, The theory of the Riemann zeta-function, Clarendon Press, Oxford, 1951, pp. 181-182, 281. MR 13, 741.

4. - The theory of functions, Clarendon Press, Oxford, 1939, pp. 44-45.

5. A. M.Vaidya, On the order of the error function of the square-free numbers, Proc. Nat. Inst. Sci. India Part A 32 (1966), 196-201. MR 40 \#2623.

Department of Mathematics, Gujarat University, Ahmedabad 9, India 